\title{
Curvatura del raquis en escolares y relación del peso de sus mochilas con el grado de incapacidad física en función del sexo \\ Spine curvature in school children and the relation between backpack weight and degree of physical disability by gender \\ *José Antonio Hernández, **María Espada-Mateos, ***Javier Abián-Vicén, ****Pablo Abián-Vicén \\ *Universidad Camilo José Cela (España), **Universidad Politécnica de Madrid (España), *** Universidad de Castilla-La Mancha
} (España), ${ }^{* * * *}$ Universidad Pontificia Comillas (España)

\begin{abstract}
Resumen. Objetivo: Analizar la morfología del raquis en escolares, comparar su forma en función del género y relacionar la aparición de anomalías con el peso de sus mochilas y el grado de incapacidad física. Material y Métodos: Se sometió a 219 escolares de entre 12 y 15 años (127 chicos y 92 chicas) a una medición de la situación de los cuerpos vertebrales y de la angulación de la columna torácica, lumbar y sacro-cadera mediante el uso del Spinal Mouse ${ }^{\circledR}$, se pesaron sus mochilas y respondieron el cuestionario de Roland-Morris. Resultados: Las chicas presentaron mayor angulación en la zona torácica con unos valores de $24.4 \pm 16.4^{\circ}$ frente a $11.4 \pm 17.9^{\circ}$ de los chicos $(p<0.05)$. No se encontraron diferencias entre chicos y chicas en la angulación de la zona lumbar, en la angulación de la zona sacro-cadera ni en la longitud de la columna vertebral. Las chicas presentaron mayor número de alteraciones en el raquis $(p<0.001)$ y un mayor peso de las mochilas ( $p=0.007)$ que los chicos. En el cuestionario de Roland-Morris no se encontraron diferencias entre el grupo de chicas (1.0 \pm 1.6 puntos) y de chicos ( $0.7 \pm 1.3$ puntos). Conclusiones: Las diferencias morfológicas encontradas entre chicos y chicas nos indican que los chicos escolares analizados presentan una rectificación de la zona torácica y que las chicas tienen una mayor curvatura entre la mayoría de las vértebras dorsales y lumbares, así como un mayor número de alteraciones en el raquis.

Palabras clave: Columna vertebral, peso de las mochilas, género, vértebras torácicas, vértebras lumbares.
\end{abstract}

\begin{abstract}
Objective: To analyze the morphology of the spine in school children, to compare spinal shape depending on gender, and to relate the appearance of anomalies with the weight of their backpacks and the degree of physical disability. Materials and methods: The position of the spinal vertebrae, as well as the thoracic, lumbar, and pelvic curvatures in standing position in 219 school children between 12 and 15 years old (127 boys and 92 girls) were measured using a Spinal Mouse ${ }^{\circledR}$. Their backpacks were weighed and the students responded the Roland-Morris questionnaire. Results: Girls showed greater values on the thoracic curvature of the spine, with scores of $24.4 \pm 16.4^{\circ}$ versus $11.4 \pm 17.9^{\circ}$ in boys $(p<0.05)$. No significant differences were found between boys and girls in the angulation of the lumbar area, in the pelvis tilt, or in the length of the spine. Girls showed more alterations in the spine $(p<0.001)$ and greater backpack weight $(p=0.007)$ than boys. No differences were found in the Roland-Morris questionnaire scores between girls ( $1.0 \pm 1.6$ points) and boys ( $0.7 \pm 1.3$ points). Conclusions: The morphological differences found between boys and girls indicate that the former present a rectification of the thoracic area, whereas the latter present a greater curvature between most of the thoracic and lumbar vertebrae, as well as a greater number of spine alterations.
\end{abstract}

Key words: Spine; backpack weight; gender; thoracic vertebrae; lumbar vertebrae.

\section{Introducción}

Existe una preocupación social creciente sobre el dolor de espalda en los adolescentes producido por el largo tiempo que se encuentran en la posición de sedestación (Murphy, Buckle, \& Stubbs, 2004), el peso que cargan en sus mochilas (Alberola, Pérez, Casares, Cano, \& Andrés, 2010; García, 2009) o el sedentarismo (Beltrán-Carrillo, Devís-Devís, \& PeiróVelert, 2012; Mikkonen et al., 2016; Noriega et al., 2015). El dolor de espalda constituye un problema fundamental de salud puesto que afecta tanto a la población infantil como a la adulta (González, Rodríguez, De La Puente, \& Díaz, 2000; Kovacs et al., 2003; Lafian \& Torralba, 2018) siendo uno de los tipos de dolor más frecuente entre niños y niñas desde los 6 a los 18 años (Calvo-Muñoz, Gómez-Conesa, \& SánchezMeca, 2012). Científicos han determinado que solo el 15.2\% de los estudiantes tienen una postura correcta (Kriventsova et al., 2017). En la población adolescente, entre 13 y 15 años, diversos autores han encontrado una prevalencia de dolor de espalda entre el 40\% y el 69\% (Jones, Watson, Silman, Symmons, \& Macfarlane, 2003; Kovacs et al., 2003; Nosko,

Fecha recepción: 20-08-18. Fecha de aceptación: 23-01-19 Pablo Abián Vicén

pabloo9@hotmail.com
Razumeyko, Iermakov, \& Yermakova, 2016; Razumeyko, 2015). En la mayoría de los casos no está clara cuál es la etiología del dolor de espalda, solamente se puede identificar una causa bien definida asociada a anormalidades clínicamente significativas en menos de un $20 \%$ de los sujetos que padecen este dolor (García, 2009; Spoor \& Öner, 2013). En el resto de los casos la etiología resulta desconocida (Pérez Guisado, 2006), dando lugar a un dolor inespecífico, aunque diversos autores proponen como origen de la mayoría de los casos de dolor de espalda y alteraciones posturales, la retracción crónica de los músculos que componen la gran cadena muscular posterior, la cual es la encargada de mantenernos en postura bípeda frente a la gravedad (Busquet, 1999; Denys-Struyf \& Girons, 2001). Esta retracción muscular sería debida a la solicitación constante de la musculatura posterior para contraponerse a la gravedad (Denys-Struyf \& Girons, 2001).

A raíz de esta tendencia que tiene la musculatura a fibrotizarse y retraerse (Souchard \& Ollier, 2002), se plantea que las articulaciones deben moverse fuera de sus recorridos fisiológicos con lo cual los músculos se contraen y acortan de forma crónica pudiendo ser éste el origen del dolor (Liebenson, 2002). También ha sido planteado que este proceso de retracción muscular posterior altera los patrones de movimiento fisiológico de las articulaciones sobre las que actúa, pudiendo también ocasionar la aparición de dolor 
(Denys-Struyf \& Girons, 2001). Algunos autores han relacionado las alteraciones morfológicas en el raquis con la aparición de dolor de espalda (Iwamoto, Abe, Tsukimura, \& Wakano, 2004; Spoor \& Öner, 2013).

La hipercifosis es un aumento de la curvatura dorsal que se da de forma frecuente en la adolescencia, estando la medición normal entre $20^{\circ}$ y $45^{\circ}$, si pasa de $45^{\circ}$ se diagnostica como hipercifosis (leve hipercifosis torácica de $46^{\circ}$ a $60^{\circ}$ y moderada hipercifosis torácica $>60^{\circ}$ ). Un valor menor de $20^{\circ}$ se considera rectificación torácica. La hiperlordosis lumbar es un aumento de la curvatura lumbar, que tiene unos valores normales entre $-20^{\circ} \mathrm{y}-40^{\circ}$ (si es mayor de $-20^{\circ}$ se considera rectificación lumbar y si es menor de $-40^{\circ}$ hiperlordosis lumbar) (Contreras, Miranda, Ordoñez, Miranda, \& Díez, 1981; Santoja, 1993).

Varios estudios han analizado las curvaturas del raquis en diferentes poblaciones (Castillo \& Obregón, 2018; Muyor, López-Minarro, \& Alacid, 2011a, 2011b; Muyor, LópezMiñarro, \& Alacid, 2012; Muyor, Sánchez-Sánchez, SanzRivas, \& López-Minarro, 2013; Vidal, Borrás, Ponseti, Gili, \& Palou, 2010), siendo objeto de estudio varias disciplinas deportivas (ciclistas, bailarines, etc.) y en otros casos intentando relacionar el dolor de espalda de los sujetos con la práctica deportiva o el peso de las mochilas .

Los objetivos de este estudio han sido aportar valores de referencia sobre la fisionomía del raquis en adolescentes españoles y establecer si existe alguna diferencia en la morfología del mismo en función del género. Por otro lado, se analizó la relación entre el peso de la mochila y las alteraciones en la columna y el grado de incapacidad física que provoca el dolor de espalda en los adolescentes.

\section{Materiales y métodos}

\section{Sujetos}

Un total de 219 alumnos españoles, con edades comprendidas entre los 12 y los 15 años participaron voluntariamente en este estudio, de los cuales 127 fueron chicos y 92 fueron chicas. Se planteó la investigación como un estudio de campo en Institutos de Educación Secundaria Obligatoria de carácter público de la Comunidad de Madrid.

En la Tabla 1 se muestran las variables descriptivas de los sujetos. Los criterios de incursión de los alumnos para participar en el estudio fueron; (1) pertenecer a un instituto de Educación Secundaria de carácter público, (2) tener autorización paterna, (3) disposición personal para realizar la valoración y (4) no tener ninguna lesión incapacitante que no permitiese llevar a cabo la medición. Se escribió una carta a los padres informando de la prueba a realizar y solicitando su consentimiento para la misma; en ella, además de explicar cómo se iba a realizar la prueba, se les dio una breve explicación de cuál era el objetivo de la misma y de los posibles riesgos derivados de las mediciones que se iban a realizar. Más del 95\% de los padres que fueron informados autorizaron a sus hijos a realizar la valoración. El estudio cumplía

Tabla 1:

\begin{tabular}{lcccccc}
\multicolumn{7}{c}{ Variables descriptivas de la muestra analizada. } \\
\hline & Chicos & Chicas & $\Delta$ & IC $95 \%$ & p-valor & $d$ \\
\hline $\mathrm{n}$ & 127 & 93 & - & - & - & \\
Masa $(\mathrm{Kg})$ & $55.7 \pm 12.9$ & $50.7 \pm 8.1$ & $9.0 \%$ & 2.2 a 7.8 & $\mathbf{0 . 0 0 1}$ & 0.5 \\
Talla $(\mathrm{cm})$ & $163.7 \pm 9.0$ & $158.8 \pm 6.5$ & $3.0 \%$ & 2.7 a 7.1 & $\mathbf{P}<\mathbf{0 . 0 0 1}$ & 0.6 \\
I.M.C. $\left(\mathrm{Kg} / \mathrm{m}^{2}\right)$ & $20.6 \pm 3.4$ & $20.1 \pm 2.7$ & $2.4 \%$ & -0.3 a 1.3 & 0.231 & 0.1 \\
Edad $(\mathrm{annos})$ & $13.6 \pm 1.1$ & $13.3 \pm 0.9$ & $2.2 \%$ & -0.1 a 0.5 & 0.083 & 0.3 \\
\hline
\end{tabular}

todos los criterios de la última versión de la Declaración de Helsinki sobre principios éticos en investigación con seres humanos.

\section{Materiales}

La estatura y la masa corporal se obtuvieron a través de una báscula con equipo de tallado homologado (Modelo SECA 700 . Sensibilidad de \pm 50 g para la masa, $\pm 1 \mathrm{~mm}$ para la talla). El índice de masa corporal (IMC) se obtuvo posteriormente a partir de los parámetros de la talla y la masa. La fórmula para la obtención del IMC fue masa/estatura² ${ }^{2}$ expresado en unidades de $\mathrm{Kg} / \mathrm{m}^{2}$. Estos resultados fueron contrastados con las curvas y tablas de crecimiento de Sobradillo (2004).

Para la medición de la morfología del raquis se utilizó el Spinal Mouse ${ }^{\circledR}$ un sistema electromecánico computerizado no invasivo, que es utilizado para medir la curvatura espinal en diversas posturas. El aparato se pasó por toda la columna vertebral de los alumnos desde el final de la zona cervical al sacro. El Spinal Mouse ${ }^{\circledR}$ nos da una visión de las vértebras de la zona torácica, lumbar y sacra. Cuando una referencia muestra un valor positivo, nos está describiendo una relación cifótica expresada en grados, cuando nos muestra un valor negativo, la relación será lordótica (Figura 1).

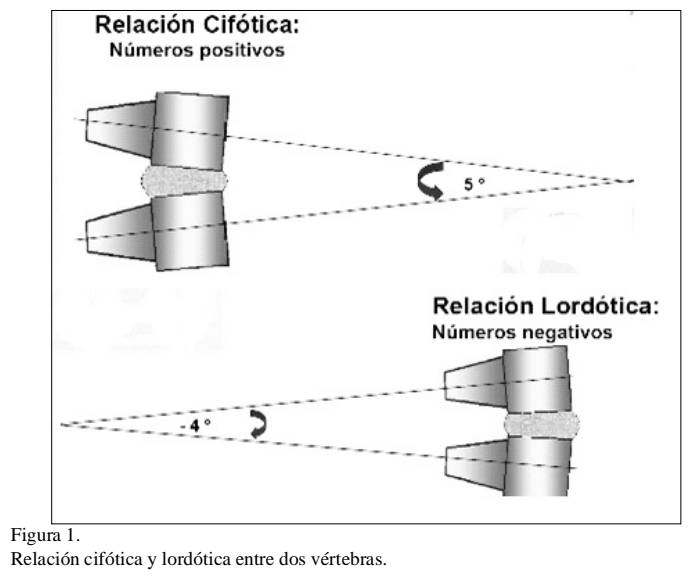

Cuando un valor se encuentra por encima de los estándares normalizados diremos que existe un exceso de movilidad o que se da una flexibilización del raquis y cuando el valor se encuentre por debajo lo denominaremos como un déficit de movilidad o rectificación (Hernández-Corvo, 1989). La validez y reproducibilidad de este instrumento ha sido analizada previamente en diversos estudios (Guermazi et al., 2006; Mannion, Knecht, Balaban, Dvorak, \& Grob, 2004).

El peso de las mochilas se obtuvo con la misma báscula que se utilizó para el pesaje y tallado de los alumnos. Se les indicó que debían traer a la prueba la mochila tal y como la habían llevado a la escuela ese mismo día.

El cuestionario utilizado para determinar el grado de incapacidad física derivado de la lumbalgia inespecífica fue el Roland Morris (Kovacs et al., 2002; Roland \& Fairbank, 2000). Este cuestionario fue completado el mismo día que se realizó la valoración con el Spinal Mouse ${ }^{\circledR}$ para lo cual, se pidió autorización a los profesores para que los alumnos pudieran rellenar el cuestionario en el aula. El cuestionario RolandMorris es un cuestionario simple de 24 preguntas sobre el dolor de espalda. El encuestado debe marcar los ítems que 
hacen referencia al grado de incapacidad que el sujeto está padeciendo el mismo día de la realización del test. Se realizó una categorización funcional en tres niveles de los datos obtenidos con el cuestionario, siguiendo la clasificación que utiliza la OMS (Organización Mundial de la Salud) en su escala de dolor (leve, moderado y severo).

\section{Protocolo de toma de datos}

El protocolo para la obtención de datos fue el siguiente: el evaluador siempre se encontraba acompañado de otro profesor o de algún miembro de la comunidad educativa (jefe de estudios, etc.). Los sujetos debían traer un bañador, en el caso de las chicas se les solicitaba que el bañador fuera de los que tienen cinta de cuello (que no se abrochase por la espalda). No se permitió el uso de collares. Tampoco se permitió el uso de relojes o algún tipo de carga en los miembros superiores que pudiese derivar en un sesgo escoliótico. El proceso para la obtención de los datos fue siempre realizado por la misma persona con una gran experiencia en mediciones antropométricas y en el uso del Spinal Mouse ${ }^{\circledR}$.

Para la medición de la talla los participantes permanecieron de pie, en posición anatómica con los talones juntos, glúteos, espalda y región occipital pegados a la barra. Los talones debían estar siempre en contacto con el tallímetro. La medición se realizó después de una inspiración profunda, con la cabeza en el plano de Frankfort. Para la medición de la masa corporal, los alumnos se desprendieron de la ropa (quedándose en bañador), así como cualquier complemento que pudiesen portar (gafas, gorro de baño etc.).

Para la medición con el Spinal Mouse ${ }^{\circledR}$ se utilizó la llamada posición neutra, el sujeto se colocó en una posición erguida; con pies separados a la anchura de la cadera, brazos estirados a los lados del cuerpo y mirada al frente.

Se identificó mediante palpación y se marcó con un rotulador dermográfico la apófisis espinosa de C7 (punto de partida) y la parte superior del pliegue interglúteo (punto final). El Spinal Mouse ${ }^{\circledR}$ fue guiado a través de la línea media de la espina empezando en la vértebra C7 y guiándolo hasta la zona del sacro, coincidiendo con el pliegue interglúteo (aproximadamente S3). Durante la medición dos ruedas giratorias siguen el contorno de la espina y los valores de angulación y distancia son enviados a un ordenador situado en las proximidades, dando una frecuencia de escaneo de 150 Hz. El tiempo de medición estimado es de 2 a 4 segundos. Se utiliza un algoritmo recursivo inteligente para calcular la posición relativa del sacro y de los cuerpos vertebrales.

\section{Variables}

Se utilizó como variable de agrupación el sexo.

En cuanto a las variables descriptivas, se incluyeron: la edad (años), la estatura (expresada en cm), la masa (expresada en $\mathrm{Kg}$ ) y el IMC (expresado en $\mathrm{Kg} / \mathrm{m}^{2}$ ).

Por último, en lo referente a las variables dependientes se registraron: el índice de curvatura intervertebral para cada vertebra, desde la T1/T2 hasta L5/S1 (expresado en grados), el índice de curvatura acumulada del conjunto de la columna torácica y del conjunto de la columna lumbar (en grados), la inclinación de las vértebras del sacro-cadera (en grados), la longitud total de la columna (expresado en cm), la inclinación del total de la columna (expresada en grados), el peso de la mochila (en Kg), el Cuestionario Roland-Morris (baremado en una escala de dolor de 24 rangos) y, por último, el número de alteraciones de espalda encontradas con el Spinal Mouse ${ }^{\circledR}$.

\section{Análisis de datos}

Se usaron los siguientes programas informáticos: Hoja de cálculo Microsoft Excel (Microsoft, España) para almacenar los resultados y el programa SPSS v. 19,0 (SPSS Inc., EE.UU) para realizar los cálculos estadísticos. Se utilizaron pruebas de estadística descriptiva y de estadística inferencial y se realizaron correlaciones de Pearson por el método de los cuadrados. Se hallaron medias, desviaciones típicas y rangos. La normalidad de cada variable fue testada inicialmente con la prueba de Kolmogorov-Smirnov, todas las variables continuas incluidas en el estudio presentaron una distribución normal ( $p>0.05)$. Como prueba inferencial para analizar las diferencias entre chicos y chicas se utilizó una prueba $t$ de Student para muestras independientes. Se usó el criterio estadístico de significación de $p<0.05$.

\section{Resultados}

En la Tabla 2 se muestran las medias y las desviaciones estándar de los grados de angulación entre los pares de vértebras desde T1 hasta S1. Se han encontrado diferencias significativas en las primeras vertebras torácicas desde T2 hasta T5, donde las chicas obtuvieron valores más cifóticos que los chicos y en las vértebras torácicas desde T10 hasta L1 y lumbares desde L1 hasta L3 donde las chicas obtuvieron valores más lordóticos que los chicos. En las vértebras L4-L5 y L5-S1 los chicos obtuvieron unos valores más lordóticos que las chicas.

El grupo de las chicas obtuvo una mayor angulación de la columna torácica $\left(24.4 \pm 16.4^{\circ}\right.$ vs $\left.11.4 \pm 17.9^{\circ}, p<0.001\right)$, un mayor número de alteraciones en la columna (6.1 \pm 2.2 vs 4.14 $\pm 1.9, p<0.001)$. y un mayor peso de las mochilas que los chicos (10.3 \pm 2.8 vs 9.2 $\pm 2.5, p=0.007)$ (Tabla 3$)$. En referencia al cuestionario de Roland-Morris no se han encontrado diferencias significativas entre las chicas y los chicos $(1.0 \pm 1.6$ respuestas afirmativas vs. $0.7 \pm 1.3$ respuestas afirmativas) (Tabla 3).

\begin{tabular}{|c|c|c|c|c|c|c|c|c|}
\hline \\
\hline & Chicos & \multicolumn{2}{|c|}{ Chicas } & \multicolumn{2}{|c|}{$\Delta$} & IC 95\% & p-valor & $d$ \\
\hline T1-T2 $\left(^{\circ}\right)$ & $-11.9 \pm 7.3$ & \multicolumn{2}{|c|}{$-12.3+8.0$} & \multicolumn{2}{|c|}{$3.4 \%$} & -1.6 a 2.5 & 0.700 & -0.1 \\
\hline T2-T3 $\left(^{\circ}\right)$ & $-8.8 \pm 7.5$ & \multicolumn{2}{|c|}{$-4.2 \pm 9.6$} & \multicolumn{2}{|c|}{$-53.4 \%$} & $\begin{array}{l}-1.0 \mathrm{~d} 2.5 \\
-7.0 \mathrm{a}-2.3\end{array}$ & $\mathbf{P}<\mathbf{0 . 0 0 1}$ & 0.5 \\
\hline T3-T4 $\left(^{\circ}\right)$ & $-5.7 \pm 9.3$ & \multicolumn{2}{|c|}{$3.9 \pm 8.5$} & \multicolumn{2}{|c|}{$170.2 \%$} & -12.1 a -7.3 & $P<0.001$ & -1.1 \\
\hline T4-T5 $\left(^{\circ}\right)$ & $0.0 \pm 8.3$ & \multirow{2}{*}{\multicolumn{2}{|c|}{$\begin{array}{l}5.0 \pm 6.7 \\
4.946\end{array}$}} & \multicolumn{2}{|c|}{$500 \%$} & $\begin{array}{l}-7.0 \mathrm{a}-2.9 \\
-1\end{array}$ & $\mathbf{P}<0.001$ & -0.7 \\
\hline T5-T6 $\left(^{\circ}\right)$ & $4.0 \pm 5.0$ & & & \multirow{2}{*}{\multicolumn{2}{|c|}{$\begin{array}{c}-22.5 \% \\
14.3 \%\end{array}$}} & -2.2 a 0.4 & 0.178 & -0.2 \\
\hline T6-T7 $\left({ }^{\circ}\right)$ & $6.3 \pm 3.6$ & \multicolumn{2}{|c|}{$5.4 \pm 4.8$} & & & \multirow{2}{*}{-0.2 a 2.0} & \multirow{2}{*}{0.112} & 0.2 \\
\hline T7-T8 $\left(^{\circ}\right)$ & $6.7 \pm 3.5$ & \multicolumn{2}{|c|}{$\begin{array}{l}3.4 \pm 4.8 \\
6.5 \pm 6.4\end{array}$} & \multicolumn{2}{|c|}{$\begin{array}{l}14.5 \% \\
4.5 \%\end{array}$} & & & 0.0 \\
\hline T8-T9 $\left(^{\circ}\right)$ & $6.5 \pm 3.1$ & \multicolumn{2}{|c|}{$6.7 \pm 6.1$} & & $1 \%$ & -1.6 a 1.1 & 0.734 & 0.0 \\
\hline T9-T10 $\left({ }^{\circ}\right)$ & $7.1 \pm 3.9$ & & $2 \pm 7.0$ & & $.7 \%$ & -0.6 a 2.5 & 0.241 & 0,2 \\
\hline T10-T11 ( $\left.{ }^{\circ}\right)$ & $5.3 \pm 4.1$ & & $6 \pm 7.6$ & & $1 \%$ & 0.1 a 3.2 & 0.040 & 0.3 \\
\hline T11-T12 $\left(^{\circ}\right)$ & $2.3 \pm 3.8$ & & $1 \pm 7.1$ & & $3 \%$ & 1.7 a 4.9 & $P<0.001$ & 0.6 \\
\hline T12-L1 $\left({ }^{\circ}\right)$ & $-1.1 \pm 3.2$ & & $4 \pm 5.9$ & -20 & $9.1 \%$ & 0.9 a 3.7 & 0.001 & 0.5 \\
\hline L1-L2 $\left(^{\circ}\right)$ & $-3.5 \pm 4.3$ & & $8 \pm 4.4$ & & $.3 \%$ & 2.1 a 4.4 & $\mathrm{P}<0.001$ & 0.8 \\
\hline L2-L3 $\left(^{\circ}\right)$ & $-8.0 \pm 4.5$ & & $.6 \pm 5.5$ & & $.8 \%$ & 2.2 a 4.9 & $\mathbf{P}<0.001$ & 0.7 \\
\hline L3-L4 $\left(^{\circ}\right)$ & $-9.9 \pm 4.7$ & & $9 \pm 6.9$ & & & -2.5 a 0.8 & 0.291 & -0.2 \\
\hline L4-L5 ( $\left(^{\circ}\right)$ & $-7.4 \pm 8.4$ & & $9 \pm 9.3$ & & $8 \%$ & -4.9 a -0.2 & 0.034 & -0.3 \\
\hline L5-S1 $\left(^{\circ}\right)$ & $-3.1 \pm 9.6$ & & $2 \pm 8.6$ & & $.5 \%$ & -5.7 a -0.8 & 0.009 & -0.4 \\
\hline $\begin{array}{l}\text { Tabla } 3 . \\
\text { Curvaturas acum } \\
\text { Morris. }\end{array}$ & 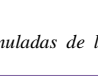 & & li. & & & & & \\
\hline & Chicos & & Chic & & $\Delta$ & IC $95 \%$ & $\mathrm{p}$-valor & $d$ \\
\hline Torácica $\left({ }^{\circ}\right)$ & $11.4 \pm 1 ?$ & & $24.4 \pm$ & & $-113.2 \%$ & $\%-17.5$ a -8.3 & $3 \quad \mathbf{P}<\mathbf{0 . 0 0 1}$ & -0.8 \\
\hline Lumbar $\left({ }^{\circ}\right)$ & $-32.9 \pm \mathrm{c}$ & & $-35.3 \pm$ & 10.6 & $-7.3 \%$ & $\quad-0.3$ a 5.1 & 0.086 & 0.2 \\
\hline Sacro-Cadera $\left({ }^{\circ}\right)$ & $22.8 \pm 8$ & & $22.8 \pm$ & & $0 \%$ & -2.3 a 2.3 & 0.992 & 0 \\
\hline Inclinación $\left({ }^{\circ}\right)$ & $7.8 \pm 3$. & & $6.9 \pm$ & & $11.5 \%$ & 0.2 a 1.7 & 0.018 & 0.3 \\
\hline Longitud (cm) & $539.1 \pm 5$ & & $527.3 \pm$ & & $2.2 \%$ & $6 \quad-2.0$ a 25.5 & 0.095 & 0.2 \\
\hline $\begin{array}{l}\text { Alteraciones } \\
\text { Columna }\left(\mathrm{n}^{\circ}\right)\end{array}$ & $4.14 \pm 1$ & & $6.1 \pm$ & & $-45.9 \%$ & $\% \quad-2.5$ a -1.4 & $\mathrm{P}<0.001$ & -1 \\
\hline Peso mochila $(\mathrm{Kg})$ & g) $9.2 \pm 2$. & & 10.3 & & $-12 \%$ & -1.9 a -0.3 & 0.007 & -0.4 \\
\hline Roland-Morris & $0.7 \pm 1$. & & $1.0 \pm$ & & $-42.9 \%$ & $\% \quad-0.8$ a 0.1 & 0.183 & -0.2 \\
\hline
\end{tabular}


Se obtuvieron correlaciones no significativas entre el número de alteraciones de la columna y el grado de incapacidad física ( $r=0.135 ; p=0.082$ ) y entre el peso de la mochila y el grado de incapacidad física $(r=0.155 ; p=0.064)$.

\section{Discusión}

Los principales resultados de este estudio mostraron una diferencia significativa en la angulación de la columna torácica, en el número de alteraciones de la columna y en el peso de las mochilas entre los chicos y las chicas. El grupo de las chicas presentó una mayor angulación en las diferentes curvas del raquis además de un mayor número de alteraciones en las inclinaciones vertebrales. Por otro lado, los chicos presentaron una rectificación de la columna torácica. Las diferencias significativas observadas entre el grupo de chicos y chicas, al igual que el alto peso que llevan en sus mochilas deberían ser tenidas en cuenta para intentar corregir ciertas posturas que se llevan a cabo en la vida escolar y cotidiana, para intentar reducir el peso de las mochilas y evitar la incapacidad física y el dolor de espalda que puede convertirse en un problema más grave con el paso del tiempo.

Se observó que la angulación de las primeras vertebras torácicas (T2 a T5) tuvieron una angulación más cifótica en el grupo de las chicas. Esto podría ser debido al mayor peso que tiene en las chicas el pecho, lo que puede incidir en que desarrollen una posición más encorvada (cifótica). En la curvatura acumulada de la columna torácica los chicos han obtenidos unos valores de $11.4 \pm 17.9^{\circ}$, y las chicas de $24.4 \pm$ $16.4^{\circ}$, lo que significa un $113.2 \%$ más de curvatura las chicas. Los valores de las chicas están dentro de la normalidad de los valores de referencia aportados por Contreras et al. (1981) y Santoja (1993), mientras que los valores de los chicos están por debajo, presentando una rectificación torácica. Al comparar nuestros resultados con otros estudios podemos observar como los valores aportados por Castillo and Obregón (2018) en su estudio con bailarines españoles son superiores para la columna torácica $\left(34.18 \pm 5.10^{\circ}\right)$ que los de los escolares analizados en nuestro estudio. Muyor et al. (2011a) en un estudio sobre la curvatura de la columna torácica con un grupo de ciclistas de élite y con un grupo de personas sedentarias obtuvo unos valores de $48.17 \pm 8.05^{\circ}$ y de $42.68 \pm 8.02^{\circ}$, respectivamente. En la misma línea Muyor et al. (2012) realizó otro estudio, esta vez con ciclistas de élite y máster 30 obteniendo unos datos para la curvatura de la columna torácica por encima de $40^{\circ}$ (élite: $47.96 \pm 7.23^{\circ} \mathrm{y}$ máster 30: $47.82 \pm 9.32^{\circ}$ ), lo que demuestra que el hacer el deporte del ciclismo provoca en esos sujetos una hipercifosis torácica. Un estudio con tenistas adolescentes aportó datos para la curvatura de la columna torácica de $43.83 \pm 7.87^{\circ}$ en los chicos y $36.13 \pm 6.69^{\circ}$ en las chicas (Muyor et al., 2013).

Algunos aspectos que también pueden incidir en estas diferencias en la angulación de la columna torácica son la mayor masa muscular de los chicos frente a las chicas en estas edades (Alonso, Carranza, Rueda, \& Naranjo, 2014) y la mayor práctica de actividad física de los chicos (Muyor et al., 2013), ya que pueden tener incidencia en que la musculatura dorsal de los chicos sea más fuerte y les lleve a rectificar la curvatura de esta zona y a adquirir una posición más ergui- da. Aunque hay controversia entre los autores respecto a la relación entre la práctica deportiva y el dolor de espalda. Algunos autores afirman que hacer deporte (más de 1 hora por semana) aumenta las probabilidades de sufrir dolor de espalda (Kamada et al., 2016; Sato et al., 2011). Por el contrario, Mogesen, Gausel, Wdderkopp, Kjaer, and Leboeuf-Y de (2007) afirman que no hay relación entre hacer deporte y el dolor de espalda. Lo que sí está claro es que algunos factores como el tiempo empleado en hacer deporte y el tipo de deporte influyen en la relación deporte-dolor de espalda (Balagué, Troussier, \& Salminen, 1999) y en la morfología de la columna vertebral.

En referencia a la curvatura de la columna lumbar no se han encontrado diferencias significativas entre los chicos y las chicas. Los valores mostrados tanto para los chicos ($\left.32.9 \pm 9.6^{\circ}\right)$ como para las chicas $\left(-35.3 \pm 10.6^{\circ}\right)$ se encuentran dentro de los rangos normales (entre $-20 \mathrm{y}-40^{\circ}$ ) aportados por Contreras et al. (1981); Santoja (1993). Algunos autores han afirmado que las chicas presentan mayores dolores lumbares que los chicos (Calvo-Muñoz et al., 2012; Mikkonen et al., 2016). Hernández-Corvo (1989) plantea que, la mayor curvatura en un segmento de la columna, repercute en que las otras curvas vertebrales tienden a incrementarse como efecto compensatorio. En relación con este autor se encuentran nuestros resultados ya que las chicas presentan una mayor curvatura entre las vértebras T2-T5 (angulación más cifótica), entre las vértebras T10-L3 (angulación más lordótica) y entre L5-S1 (angulación más cifótica).

Los valores aportados por otros autores para la curvatura de la columna lumbar son ligeramente inferiores que los de nuestro estudio. Muyor et al. (2012) aporta unos valores para ciclistas de elite de $-27.62 \pm 6.97^{\circ}$ y para ciclistas máster 30 de -26.58 \pm 5.97 mientras que Castillo and Obregón (2018) aporta unos valores en su estudio con bailares españoles de $28.71 \pm 4.91$. Muyor et al. (2013) aporta unos valores con tenistas adolescentes de curvatura de la columna lumbar de $-27.58 \pm 7.01^{\circ}$ en los chicos y de $-32.69 \pm 5.06^{\circ}$ en las chicas. Según estos datos puede ser que la práctica del tenis en adolescentes ayude a tener una mayor curvatura de la columna lumbar.

Cuando comparamos el número de alteraciones de la columna vertebral en los chicos con las chicas, encontramos diferencias significativas. Las chicas presentaron un $45.9 \%$ más de alteraciones (chicos: $4.14 \pm 1.9$ vs chicas: $6.1 \pm 2.2$, $p<0.001$ ). Estos datos contrastan con la etapa de mayor variabilidad en la que se encuentran los chicos. Sobradillo (2004) nos presenta unas curvas de crecimiento donde los mayores incrementos de estatura en los chicos se dan entre los 13 años $(7.22 \mathrm{~cm})$ y los 15 años $(7.33 \mathrm{~cm})$, mientras que en las chicas se dan entre los 11 años $(6.62 \mathrm{~cm})$ y los $12(7.24 \mathrm{~cm})$.

Se encontraron diferencias significativas en el peso de las mochilas entre los chicos y las chicas. El peso de la mochila fue un $12.0 \%$ mayor en las chicas que en los chicos (chicas: $10.3 \pm 2.8 \mathrm{~kg}$ vs chicos: $9.2 \pm 2.5 \mathrm{~kg}, p=0.007$ ). Hay varios estudios que relacionan el peso de las mochilas con el dolor de espalda de los adolescentes y varios autores están en la línea de nuestro estudio y no han encontrado relación entre el peso de las mochilas y el dolor de espalda o el grado de incapacidad (Alberola et al., 2010; Chacón-Borrego, Ubago-Jiménez, La Guardia García, Padial Ruz, \& Cepero 
González, 2018; García, 2009), aunque concluyen afirmando que el peso de la mochila influye en la sensación de malestar en la infancia y debe considerarse un problema de salud.

En nuestro estudio no hemos encontrado correlación entre el peso de las mochilas y el grado de incapacidad física de los escolares, pero si hemos podido observar como el peso de las mochilas de los escolares españoles estudiados representa en el caso de los chicos un $16.5 \%$ y en el caso de las chicas un 20.3\% de su peso corporal. Nuestros datos son superiores que los aportados por otros autores (Alberola et al., 2010; García, 2009; Vidal et al., 2010). Alberola et al. (2010) en su estudio con 159 escolares (primaria y secundaria), presentaron un peso de la mochila de $6.3 \pm 2 \mathrm{~kg}$ (13.4\% de su peso corporal) y García (2009) con 61 escolares (primaria) presentó un peso de la mochila de $6.5 \pm 1.5 \mathrm{~kg}$. Esta diferencia de peso entre nuestro estudio y los otros autores puede ser debida a que ellos utilizaron en el estudio escolares de primaria y necesiten llevar menos cosas en las mochilas que los escolares de secundaria.

Lo que sí está claro es que los escolares analizados superan en el peso de la mochila el $10 \%$ del peso corporal y que en ciertos países (Austria, Alemania, etc.) marcan como límite (Negrini \& Carabalona, 2002; Skaggs, Early, D’Ambra, Tolo, \& Kay, 2006). En esta misma línea están otros especialistas que dicen que un peso de la mochila superior al $10 \%$ de la masa corporal del alumno supone un incremento del consumo energético, incrementa la inclinación de la columna y reduce el volumen pulmonar (Hong \& Brueggemann, 2000; Lai \& Jones, 2001). Este es un dato a tener en cuenta por todas las personas (padres, profesores, familiares, etc.) que pueden tener influencia en los adolescentes a la hora de ayudarles a hacer la mochila o a la hora de aconsejarles las cosas que deben de llevar en la mochila.

No se han encontrado diferencias significativas en el cuestionario Roland-Morris entre los chicos y las chicas. Los chicos han obtenido unos valores de $0.7 \pm 1.3$ y las chicas de $1.0 \pm 1.6$ respuestas afirmativas. Esto supone que el día del cuestionario las chicas estaban incapacitadas para realizar una actividad cotidiana como consecuencia de su dolor de espalda. Kovacs (2005) analiza los resultados obtenidos en el cuestionario y afirma que una puntuación por debajo de 4 puntos es una incapacidad muy leve. Con estos resultados podemos decir que los escolares analizados no presentan una incapacidad física y por lo tanto no tienen una limitación de las actividades cotidianas. Cabe resaltar que el cuestionario de Roland-Morris no sirve para medir la intensidad del dolor, ni siquiera de manera indirecta, puesto que dolor e incapacidad no se correlacionan bien (Kovacs, Abraira, \& Zamora, 2004).

\section{Conclusiones}

A partir de estos resultados, podemos afirmar que existen diferencias morfológicas en el raquis entre los chicos y las chicas escolares estudiados. Se han aportado valores de referencia sobre la fisionomía del raquis en escolares con edades comprendidas entre los 12 y los 15 años. La curvatura torácica $(\mathrm{p}<0.001)$, el número de alteraciones de la columna $(p<0.001)$ y el peso de las mochilas ( $p=0.007)$ fue mayor en las chicas que en los chicos. Tanto en los chicos (20.3\%) como en las chicas (16.5\%) se superó el peso de la mochila recomendado por varios autores (10\% del peso corporal).

Se observó una mayor curvatura entre la mayoría de las vértebras en las chicas que en los chicos. Entre las vértebras T2-T5 una angulación más cifótica, entre las vértebras T10L3 una angulación más lordótica y entre L5-S1 una angulación más cifótica en las chicas. Esto nos hace pensar que la mayor curvatura en una zona del raquis repercute en que otras zonas tengan también una mayor curvatura como efecto compensatorio.

Los resultados de este estudio pueden ser usados por profesionales de la salud (médicos, fisioterapeutas, enfermeros, etc.) que tenga a su alcance la posibilidad de usar el Spinal Mouse ${ }^{\circledR}$ para poder intentar una intervención temprana sobre las posibles alteraciones registradas en la columna, como para tratar de discernir la etiología detrás de la incapacidad física.

\section{Referencias}

Alberola, S., Pérez, I., Casares, I., Cano, A., \& Andrés, J. M. (2010). Mochilas escolares y dolor de espalda en la población infantil. Revista Pedriatría de Atención Primaria, 12(47), 385-397.

Alonso, F. J., Carranza, M. D., Rueda, J. D., \& Naranjo, J. (2014). Composición corporal en escolares de primaria y su relación con el hábito nutricional y la práctica reglada de actividad deportiva. Revista Andaluza de Medicina del Deporte, 7(4), 137142.

Balagué, F., Troussier, B., \& Salminen, J. J. (1999). Non-specific low back pain in children and adolescents: Risk factors. Eur Spine J, 8, 429-438.

Beltrán-Carrillo, V. J., Devís-Devís, J., \& Peiró-Velert, C. (2012). Actividad física y sedentarismo en adolescentes de la comunidad valenciana. Revista Internacional de Medicina y Ciencias de la Actividad Física y el Deporte., 12(45), 122-137.

Busquet, L. (1999). Las cadenas musculares: Lordosis. Cifosis. Escoliosis y deformaciones torácicas: Paidotribo.

Calvo-Muñoz, I., Gómez-Conesa, A., \& Sánchez-Meca, J. (2012). Prevalencia del dolor lumbar durante la infancia y la adolescencia: Una revisión sistemática. Rev Esp Salud Pública, 86, 331356.

Castillo, E., \& Obregón, R. (2018). Assessment of the sagittal spinal curvatures in dancers of Spanish dance. Journal of Human Sport and Exercise, 13(1), 129-137.

Chacón-Borrego, F., Ubago-Jiménez, J. L., La Guardia García, J. J., Padial Ruz, R., \& Cepero González, M. (2018). Educación e higiene postural en el ámbito de la Educación Física. Papel del maestro en la prevención de lesiones. Revisión sistemática. Retos, 34, 8-13.

Contreras, M. J., Miranda, J. L., Ordoñez, M. F., Miranda, M., \& Díez, F. (1981). Semiología del dorso curvo juvenil. Paper presented at the Jornada Monográfica vertebral, Madrid: Servicio de Rehabilitación, Hospital La Paz.

Denys-Struyf, G., \& Girons, N. C. (2001). El manual del mezierista: Paidotribo.

García, P. A. F. (2009). Dolor de espalda en alumnos de primaria y sus causas. Fisioterapia, 31(4), 137-142.

González, J. L., Rodríguez, J. M., De La Puente, E., \& Díaz, M. A. (2000). Tratamiento de la columna vertebral en la educación secundaria obligatoria: parte I - prevención y ejercicios poco recomendables. Revista Internacional de Medicina y Ciencias de la Actividad Física y el Deporte., 1(1), 27-48.

Guermazi, M., Ghroubi, S., Kassis, M., Jaziri, O., Keskes, H., Kessomtini, W., . . . Elleuch, M. H. (2006). [Validity and 
reliability of Spinal Mouse to assess lumbar flexion]. Annales De Readaptation Et De Medecine Physique: Revue Scientifique De La Societe Francaise De Reeducation Fonctionnelle De Readaptation Et De Medecine Physique, 49(4), 172-177.

Hernández-Corvo, R. (1989). Morfología funcional deportiva: Sistema locomotor: Paidotribo.

Hong, Y., \& Brueggemann, G. P. (2000). Changes in gait patterns in 10 -year-old boys with increasing loads when walking on a treadmill. Gait Posture, 11(3), 254-259.

Iwamoto, J., Abe, H., Tsukimura, Y., \& Wakano, K. (2004). Relationship between radiographic abnormalities of lumbar spine and incidence of low back pain in high school and college football players: a prospective study. Am J Sports Med, 32(3), 781-786.

Jones, G. T., Watson, K. D., Silman, A. J., Symmons, D. P., \& Macfarlane, G. J. (2003). Predictors of low back pain in British schoolchildren: a population-based prospective cohort study. Pediatrics, 111(4 Pt 1), 822-828.

Kamada, M., Abe, T., Kitayuguchi, J., Imamura, F., Lee, I. M., Kadowaki, M., ... Uchio, Y. (2016). Dose-response relationship between sports activity and musculoskeletal pain in adolescents. Pain, 157, 1339-1345.

Kovacs, F. M. (2005). El uso del cuestionario de Roland-Morris en los pacientes con lumbalgia asistidos en Atención Primaria. Semergen - Medicina de Familia, 31(7), 333-335.

Kovacs, F. M., Abraira, V., \& Zamora, J. (2004). Correlation between pain, disability and quality of life in patients with common low back pain. Spine, 29, 206-210.

Kovacs, F. M., Gestoso, M., Gil del Real, M. T., Lopez, J., Mufraggi, N., \& Mendez, J. I. (2003). Risk factors for non-specific low back pain in schoolchildren and their parents: a population based study. Pain, 103(3), 259-268.

Kovacs, F. M., Llobera, J., Gil Del Real, M. T., Abraira, V., Gestoso, M., Fernandez, C., \& Primaria Group, K. A. (2002). Validation of the spanish version of the Roland-Morris questionnaire. Spine (Phila Pa 1976), 27(5), 538-542.

Kriventsova, I., Pashkevych, S., Iermakov, S., Bartík, P., Michal, J., Nosko, M., \& Yermakova, T. (2017). Fitness-aerobic training of 15-17 years' age girl students, who have significant risk of deviations in backbone functional state. Journal of Human Sport and Exercise, 12(4), 1289-1297.

Lafian, A. M., \& Torralba, K. D. (2018). Lumbar spinal stenosis in older adults. Rheum Dis Clin North Am, 44(3), 501-512.

Lai, J. P., \& Jones, A. Y. (2001). The effect of shoulder-girdle loading by a school bag on lung volumes in Chinese primary school children. Early Hum Dev, 62(1), 79-86.

Liebenson, C. (2002). Manual de rehabilitación de la columna vertebral. In (2002 ed., pp. 515). Barcelona: Paidotribo.

Mannion, A. F., Knecht, K., Balaban, G., Dvorak, J., \& Grob, D. (2004). A new skin-surface device for measuring the curvature and global and segmental ranges of motion of the spine: reliability of measurements and comparison with data reviewed from the literature. European Spine Journal: Official Publication Of The European Spine Society, The European Spinal Deformity Society, And The European Section Of The Cervical Spine Research Society, 13(2), 122-136.

Mikkonen, P., Heikkala, E., Paananen, M., Remes, J., Taimela, S., Auvinen, J., \& Karppinen, J. (2016). Accumulation of psychosocial and lifestyle factors and risk of low back pain in adolescence: a cohort study. Eur Spine J, 25(2), 635-642.

Mogesen, A. M., Gausel, A. M., Wdderkopp, N., Kjaer, P., \& Leboeuf-Y de, C. (2007). Is active participation in specific sport activities linked with back pain? Scand J Med Sci Sports, 17, 680-686.

Murphy, S., Buckle, P., \& Stubbs, D. (2004). Classroom posture and self-reported back and neck pain in schoolchildren. Appl
Ergon, 35(2), 113-120. doi:10.1016/j.apergo.2004.01.001

Muyor, J. M., López-Miñarro, P. A., \& Alacid, F. (2011a). A comparison of the thoracic spine in the sagittal plane between elite cyclists and non-athlete subjects. J Back Musculoskelet Rehabil, 24(3), 129-135. doi:10.3233/BMR-2011-0286

Muyor, J. M., López-Miñarro, P. A., \& Alacid, F. (2011b). Spinal posture of thoracic and lumbar spine and pelvic tilt in highly trained cyclists. J Sports Sci Med, 10(2), 355-361.

Muyor, J. M., López-Miñarro, P. A., \& Alacid, F. (2012). Valoración del raquis torácico, lumbar e inclinación pélvica en ciclistas de categoría élite y máster 30. Apunts. Educación Física y Deportes., 2(108), 17-25.

Muyor, J. M., Sánchez-Sánchez, E., Sanz-Rivas, D., \& LópezMiñarro, P. A. (2013). Sagittal spinal morphology in highly trained adolescent tennis players. J Sports Sci Med, 12(3), 588-593.

Negrini, S., \& Carabalona, R. (2002). Backpacks on schoolchildren's perceptions of load, associations with back pain and factors determining the load., Spine, 27, 187-195.

Noriega, M. J., Jaén, P., Santamaría, P., Amigo, M. T., Antolín, O., Casuso, I., .. . De-Rufino, P. M. (2015). Hábitos sedentarios en adolescentes escolarizados en Cantabria. Retos, 27, 3-7.

Nosko, M., Razumeyko, N., Iermakov, S., \& Yermakova, T. (2016). Correction of 6 to 10-year-old schoolchildren postures using muscular-tonic imbalance indicators. Journal of Physical Education and Sport, 16(3), 988-999.

Pérez Guisado, J. (2006). Lumbalgia y ejercicio físico. Revista Internacional de Medicina y Ciencias de la Actividad Física y el Deporte., 6(24), 230-247.

Razumeyko, N. S. (2015). Early diagnosis of junior school age children's posture disorders. Pedagogics, psychology, medicalbiological problems of physical training and sports, 12, 96102.

Roland, M., \& Fairbank, J. (2000). The Roland-Morris Disability Questionnaire and the Oswestry Disability Questionnaire. Spine, 25(24), 3115-3124.

Santoja, F. (1993). Exploración clínica y radiográfica del raquis sagital. Sus correlaciones (premio SOCUMOT-91). Murcia: Secretariado de publicaciones e intercambio científico. Universidad de Murcia.

Sato, T., Ito, T., Hirano, T., Morita, O., Kikuchi, R., Endo, N., \& Tanabe, N. (2011). Low back pain in childhood and adolescence: Assessment of sports activities. Eur Spine J, 20, 94-99.

Skaggs, D. L., Early, S. D., D’Ambra, P., Tolo, V. T., \& Kay, R. M. (2006). Back pain and backpacks in school children. J Pediatr Orthop, 26, 358-363.

Sobradillo, B. (2004). Curvas y tablas de crecimiento: (estudios longitudinal y transversal): Fundación Faustino Orbegozo Eizaguirre, Instituto de Investigación sobre Crecimiento y Desarrollo.

Souchard, P., \& Ollier, M. (2002). Escoliosis: su tratamiento en fisioterapia y ortopedia: Editorial Medica Panamericana.

Spoor, A. B., \& Öner, F. C. (2013). Minimally invasive spine surgery in chronic low back pain patients. J Neurosurg Sci, 57(3), 203-218.

Vidal, J., Borrás, P. A., Ponseti, X., Gili, M., \& Palou, P. (2010). Factores de riesgo asociados al dolor de espalda en escolares de entre 10 y 12 años de Mallorca. Retos, 17, 10-14.

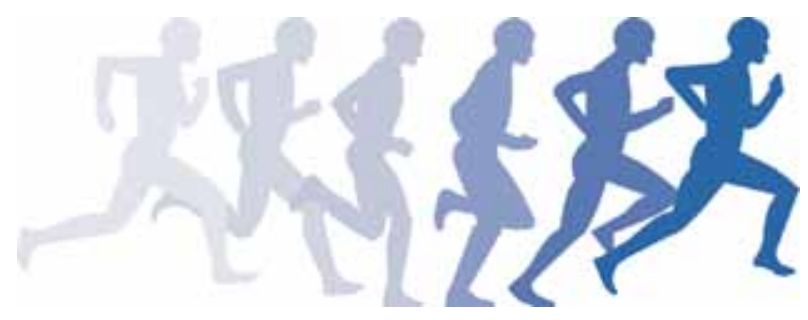

